\title{
Vulvar Cancer by AJCC v8 Stage
}

National Cancer Institute

\section{Source}

National Cancer Institute. Vulvar Cancer by A/CC v8 Stage. NCI Thesaurus. Code C139618.

A term that refers to the staging of vulvar cancer according to the American Joint Committee on Cancer, 8th edition. This staging system applies to all carcinomas of the vulva. Melanoma of the vulva is staged according to the classification for melanoma of the skin. (AJCC 8th Ed.) 